\title{
Contralateral brain atrophy in conservatively treated primary intracerebral hemorrhage
}

\section{Sung-Pil Joo, Jae-Young Kim, Yong-Hwan Cho, You-Sub Kim, Tae-Sun Kim}

Chonnam National University Hospital

\section{Brain volume loss after cerebrovascular accident}

- Cortical gray matter loss induced by neurodegenerative change

- Risk factor of neurodegenerative disease

- Suffering from dementia and cognitive defecit after minor TBI(traumatic brain injury) \& CVA

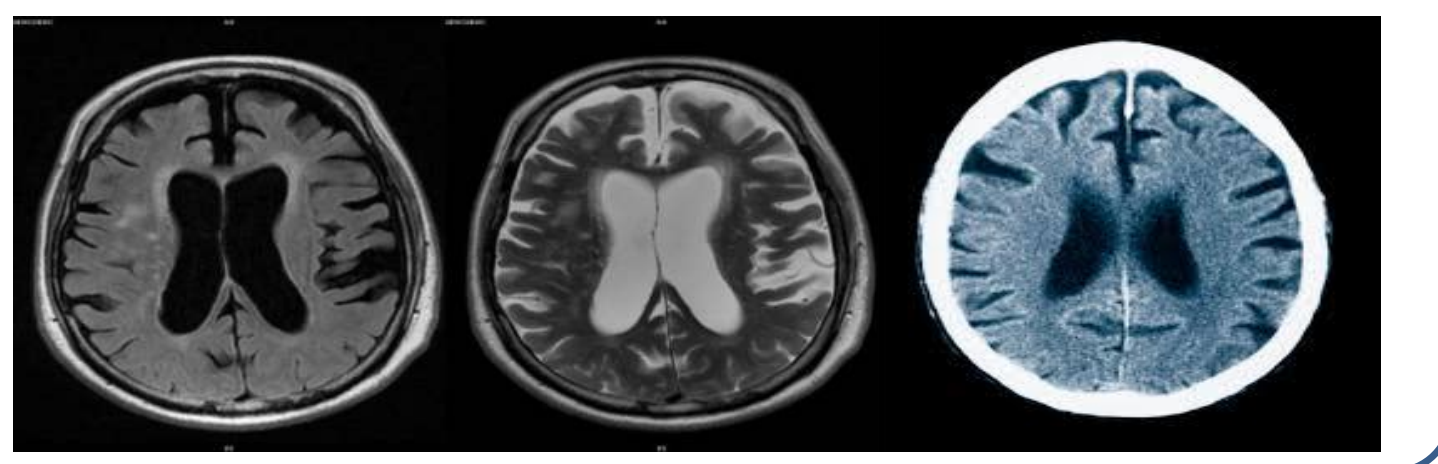

\section{Patients \& Methods}

- Total 2213 Patients

- Jan. 2010 - Dec. 2017

- primary ICH were admitted our institute

- Inclusion Criteria

Unilateral ICH

Supratentorial ICH

Presence of initial \& 3 months $~ 1$ yrs follow up brain CT scan

No evidence of surgical intervention

No History of brain disease

- 46 patients included in this study

- Serial axial images of initial and $f / u$ brain $\mathbf{C T}$ scans, respectively;

- Voxel based volume calculation

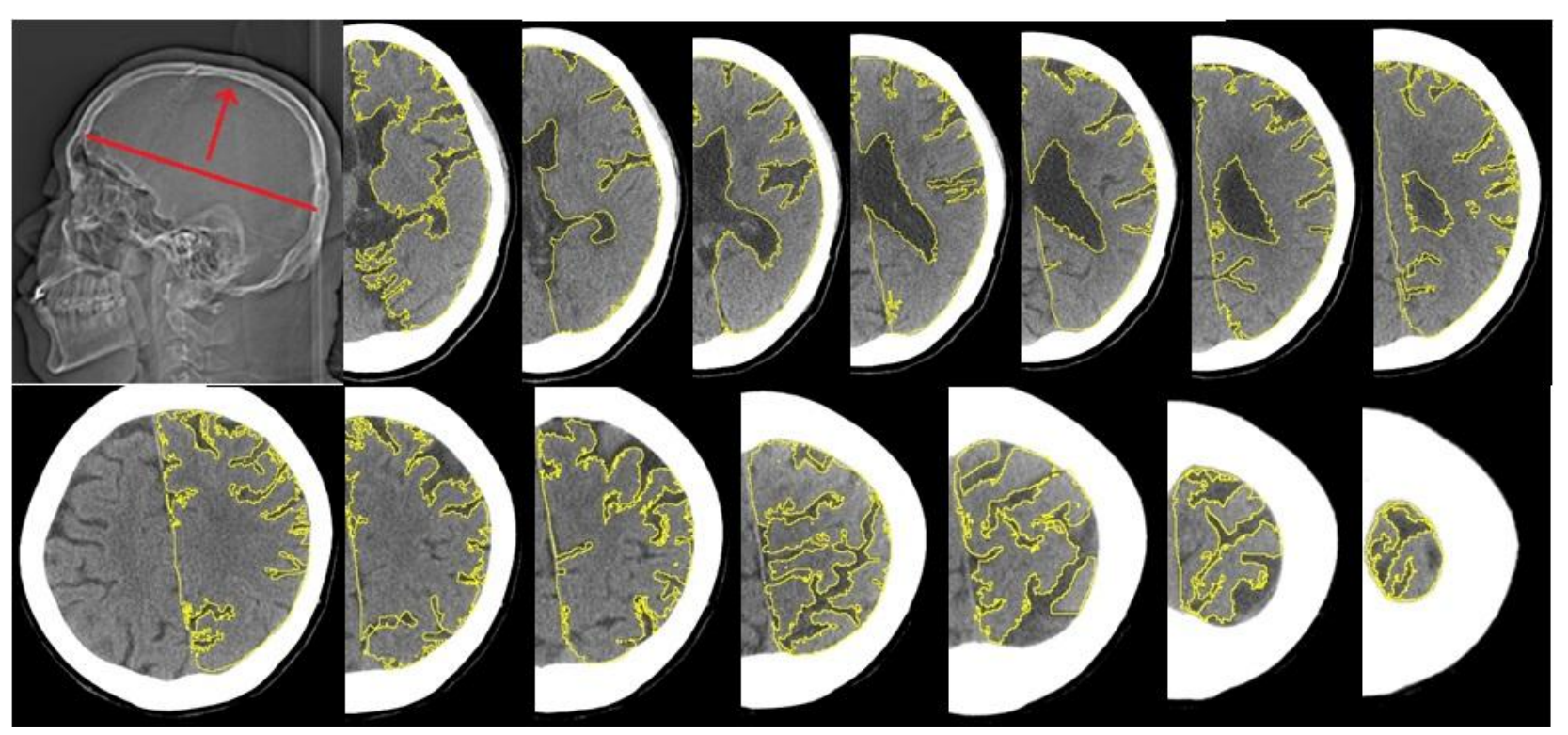

Fig. Brain volume measurement method.

Pixels counted using semi-automatic image segmentation in each plane, axial sequence image with the foramen of Monro level (horizontal red line) as the lower boundary, and the vertex (red arrow) as the upper boundary (average: approximately 14 images).

Yellow line shows the true parenchymal volume along with brain cortical margin.

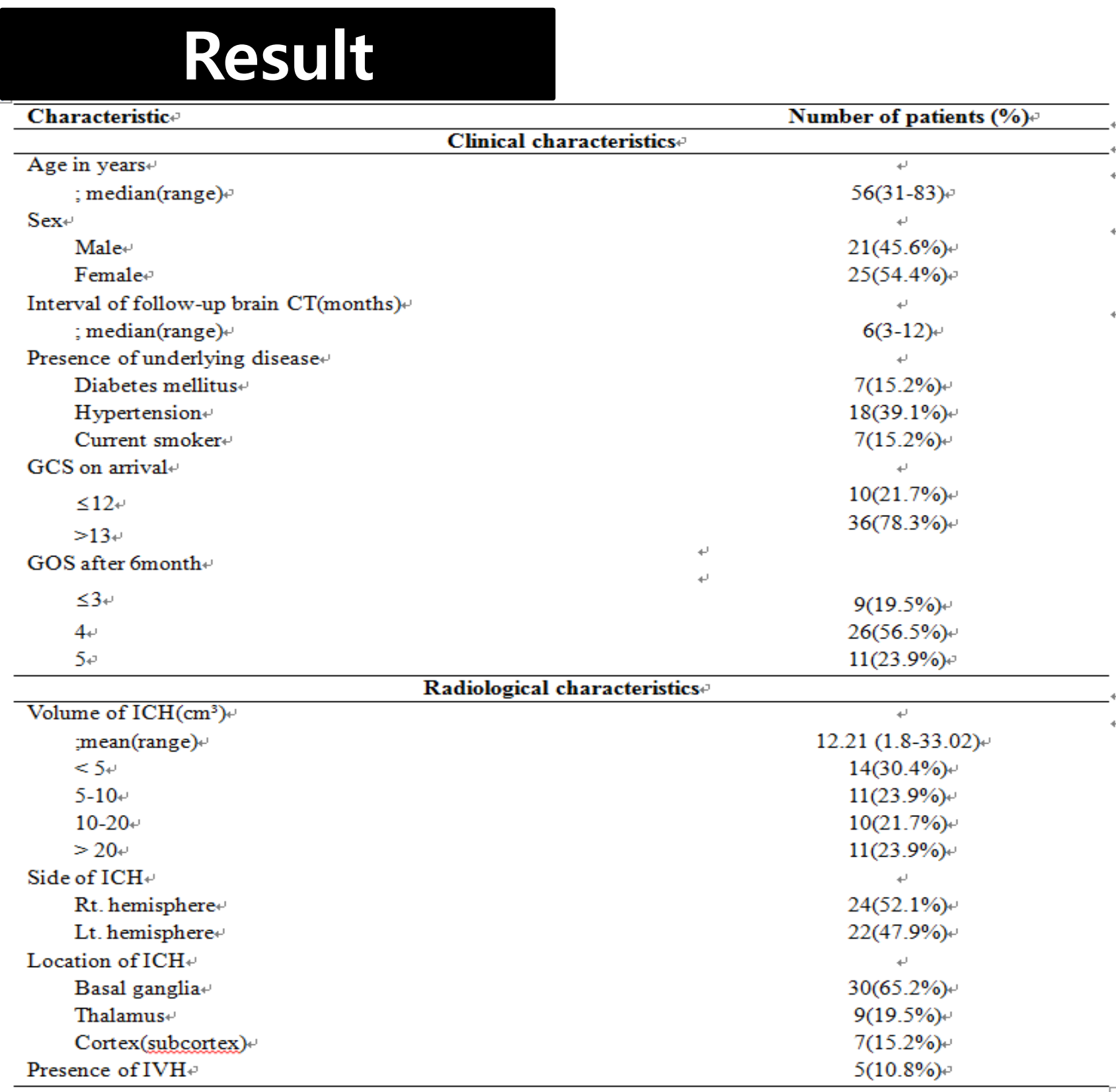

Contralateral parenchymal volume ratios at follow-up brain CT scanning

- Mean change percentage between the initial and follow-up contralateral parenchyma volume was $96.84 \%$ (range 86.08 102.63)

- The average contralateral parenchyma volume ratio decreased by $3.16 \%$ (P-value $=0.001)$.

- Normal aging process was reported to be $0.32 \%$ per year throughout all ages

- In the univariate, multivariate logistic regression model, no factors were associated with contralateral brain volume loss

\section{Discussion}

\section{Diaschisis}

- The changes of structural and functional connectivity between brain areas distant to the lesion

- Connectivity studies about the impact of a focal lesion on distant connections and its behavioural consequences.

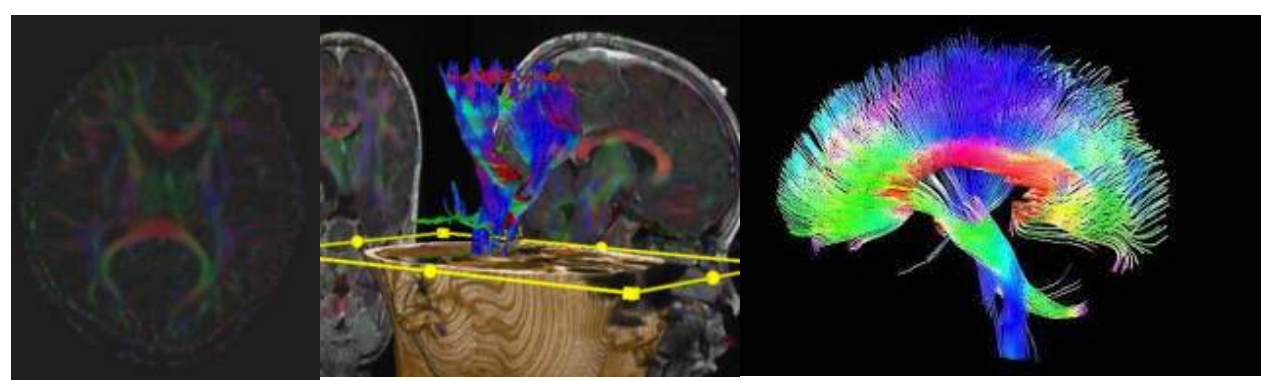

\section{Secondary neurodegeneration}

- vasogenic edema, excitotoxicity, remote atrophy, selective neuronal loss, diaschisis, and microglial activation loss

\section{CONCLUSION}

- Contralateral parenchymal volumes were significantly decreased on the brain CT scan at 1-year follow-up

- These morphological changes provided important clinical information on the remote effect of focal lesion and symptoms in the course of ICH treatment 